\title{
OPTIMUM ORIENTATION OF A MUTUALLY-SHADED GROUP OF BUILDINGS WITH RESPECT TO EXTERNAL SOLAR RADIATION
}

\author{
Meshal F. Almutairi ${ }^{1}$, Raed I. Bourisli ${ }^{1 *}$ \\ Keywords: Mutual Shading, Solar Radiation, Energy Use, Optimization
}

\begin{abstract}
External, mainly solar, load can constitute up to $80 \%$ of the energy consumed by Heating, Ventilating and AirConditioning (HVAC) systems in buildings. Proportional attention is rarely given by designers to the optimum positioning or orientation of a building with respect to the sun so as to achieve minimal solar load. Even less attention has been given to groups of buildings located within a building complex for potential mutual shading. The general practice is to place them in a row or square formation. In this work, the optimum positioning of three high-rise buildings in close proximity to each other is investigated numerically with respect to solar radiation and potential mutual shading. Specifically, the effect of the relative locations of the buildings is tested with respect to solar radiation direction for a typical summer day in Kuwait City, Kuwait. The transient three-dimensional problem is solved using the Solar Load Model of the Fluent ${ }^{\mathrm{TM}}$ finite volume computational fluid dynamics code. The solar load model calculates radiation effects from the sun's rays that enter a computational domain transiently based on the selected location. Specifically, the ray tracing approach in the model applies solar loads as heat sources in the energy equations. The solar calculator utility is used to construct the sun's location in the sky for the selected timeof-day, date, and position. As the circle containing the three buildings is rotated, the energy absorbed by the shaded one or two buildings changes significantly. Combined with air flow around the buildings, the interaction between convection and radiation heat transfer rates to the buildings can vary greatly. This variation should be taken into account when sizing HVAC equipment for the individual building. Typical TMY (Typical Meteorological Year) data for Kuwait City, Kuwait, is used to obtain boundary conditions for air velocity and temperature. Mass, momentum and energy conservation equations are solved in conjunction with the radiative Solar Load equations to obtain the combined effect. Results show that there exists an optimum orientation for the group for the selected locale and that the reduction in solar load for the optimum orientation for the group of three buildings and that the orientation effect on the total HVAC energy requirement (represented by the cooling load) is significant. The difference between the best and worst orientations was about $6 \%$. Convection, even though non-negligible, has a somewhat smaller effect on the total heat transfer and thus cooling load. Ongoing work examines the effect of the sensitivity of energy savings to wind direction and convection heat transfer, by altering strength and direction of the breeze.
\end{abstract}

\section{INTRODUCTION}

HVAC systems in hot climates are the primary consumers of electricity, with up to $70 \%$ of a country's energy output during peak load months [1]; a large part of that energy is due to direct and indirect solar heating [2, 3]. So naturally, reductions in the solar heat gain have the biggest potential in terms of reducing the HVAC system electric energy requirement. In addition to appropriate building shape, better insulation, and high thermal capacity of the building materials, proper landscaping and effective shading could also be so influential in reducing the solar load [4]. Through proper layout and orientation of a group of buildings, solar heat gain can be kept away from the buildings in the first place.

Muhaisen and Abed [5] numerically simulated the thermal performance of different orientations of buildings with different forms in Gaza Strip. They focused on buildings proportions in relation to street width, noting the effect of mutual shading they provide. Ratti et al. [6] addressed the relationship between architecture, relative compactness, shadow density, daylight distribution and openness. They reported close to $10 \%$ of potential savings in energy consumption but with little focus on optimum mutual shading configuration.

Many other researchers studied the effect of other aspect on energy consumption of many other architectural parameters and settings such as canyon geometry, such as Strømann-Andersen and Sattrup $[7,8]$. The 
energy demand of a single building in a variety of urban settings as well as the inter-play with urban canyon in terms of direct irradiation was studied by van Esch et al. [9]. The study also included analysis of potential conflict between indoor and outdoor conditions, which urban planners ought to be aware of. On the other hand, a number of other researchers studied in detail the geometry of the adjacent buildings plays in the thermal response of buildings, including buildings aspect ratios their relation to the street width. In addition to the daytime effects, the nighttime behavior with regards to cooling and natural convection were considered [10,11].

Regardless of the individual building shading device, perhaps the most imposing shading device in a group of adjacent buildings are the buildings themselves. Blocking the solar rays from hitting a building directly has a dramatic effect on reducing the solar energy intake and therefore on reducing the electric energy consumption. Reducing direct incident solar radiation also minimizes glare through glazing, which also inhibits another major source of thermal discomfort. Therefore, enough attention should be paid when laying out the footprints of proposed complex of buildings in all cases. This study focuses on a group of high-rise buildings in close proximity, possibly intended as a compound of some sort. An important key decision is of course the land lot available for the whole compound. However, with an informed decision could be made if the designers know about tradeoff between the footprint of individual buildings, the area of the whole compound, and, most importantly, the relative positions of individual towers.

\section{PROBLEM DESCRIPTION}

The transient, three-dimensional, non-isothermal, turbulent flow around three high-rise buildings located in Kuwait City, Kuwait, schematically shown in 1, is simulated to study the effect of solar radiation on the solar load absorbed by the buildings. The effect of the orientation of the buildings as a block is investigated using Fluent ${ }^{\mathrm{TM}}$ finite volume CFD software. The flow is solved using the RNG version of the two-equation $k-\varepsilon$ turbulence model. The Rosseland radiation model is used to include the radiation effect from the adjacent surfaces, to be used in the energy equation.

In order to simulate the solar radiation effect throughout the day, FLUENT solar load model was used to calculate radiation from the sun's rays that enter the computational domain. This solar ray tracing model accounts for the solar load by computing heat fluxes and applying them as a heat source in the energy equation. The surfaces of the buildings are assumed to be opaque with direct and IR absorptivity of 0.8 . The scatteral fraction, which is the amount of non-absorbed radiation that will be distributed (uniformly) across all participating surfaces, is assumed 0.5 . The setting of this parameter is required because the solar load model does not track the rays beyond the first opaque surface. The spectral fraction is assumed 0.5; this defines the split of visible and infrared (shortwave and longwave, respectively) radiation; specifically, it specifies the fraction of the direct irradiation flux that is in the visible band.

$\mathrm{Gambit}^{\mathrm{TM}}$ is used to create the geometry of the domain. As seen in figure 1, the geometry consists of three buildings with normalized height of 10 and width of 1 . As per the objective of the study, the buildings are not inline, rather, one of them (Building 1) has an offset of 0.3 in the $x$-axis direction, measured orthogonally from the adjacent faces of the two other buildings. Building 2 and 3 are set apart from Building 1 by 0.3 , measured orthogonally from the adjacent face of Building 1 . The hemispherical domain extends for about 30 units in all directions. The north is the negative $x$-direction.

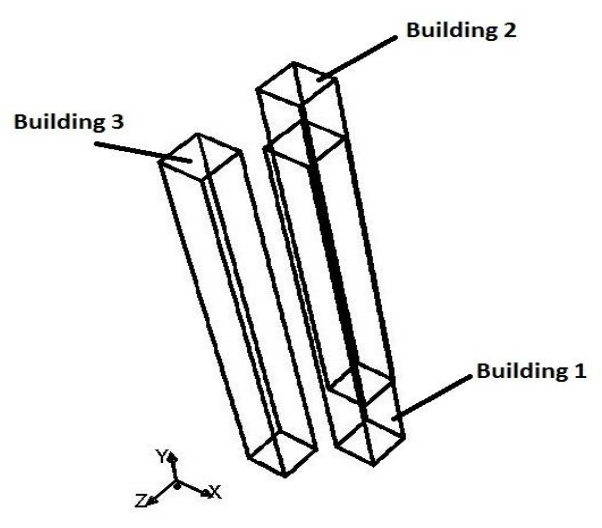

Figure 1. Schematic diagram of the problem. 


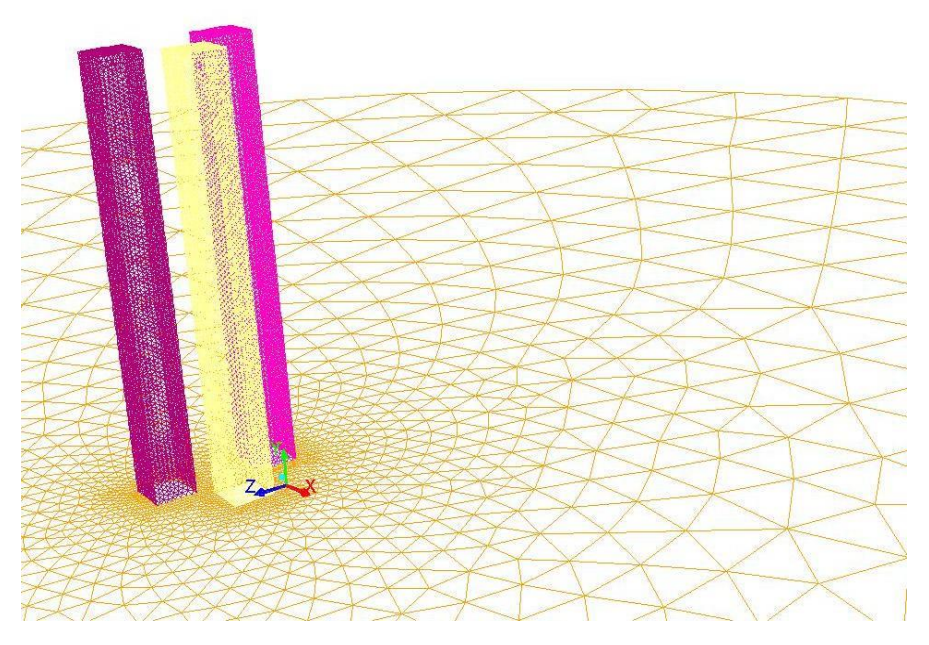

Figure 2. Sample mesh of one of the cases

The geometry is constructed using the of the Gambit ${ }^{\mathrm{TM}}$ preprocessing tool, and meshed using the TGrid feature, with cells ranging from 0.1 at the surfaces of buildings to 5 at the boundary of the domain. The mesh consists primarily of tetrahedral elements but also contained hexahedral, pyramidal, and wedge mesh elements, depending on the locations of the cells. An appropriate sizing function, with growth factor of 1.2, was used to ensure consistency and graduation of cells throughout the domain. A sample mesh is given in figure 2 .

In the Solar Ray Tracing model of the solar load model, the sun direction vector, direct solar irradiation and diffuse solar irradiation are computed using the ASHRAE Fair Weather Conditions method. For the Solar Ray Tracing model, input for Kuwait City, Kuwait, were used: global position (latitude $=29$ North, longitude $=47$ East, and time zone $=+3$ GMT). The model inputs also include the starting date and time, mesh orientation and sunshine factor, which were set to July 21, from 5:00 AM to 7:00 PM (14 hours). The sunshine factor is a linear reduction factor for the computed incident load that allows for cloud cover to be accounted for.

Fluent ${ }^{\mathrm{TM}}$ is used to obtain the finite volume solution of the governing equations. The SIMPLE algorithm was used to provide the pressure-velocity coupling. Second-order upwind discretization scheme is used for pressure, momentum and energy equations. A relatively low under-relaxation factor (of 0.1 ) for the energy equation was used to account for the complexity of introducing solar radiation as a heat source in energy equation and to allow time for the solar load interaction to reach equilibrium with other energy flow and energy quantities. The under-relaxation factors for pressure and momentum were 0.2 and 0.4 , respectively. The convergence criterion for all equations was set to $10^{-5}$ except the energy equation and the two transport equations of the $k-\varepsilon$ turbulence model which were set to $10^{-8}$ and $10^{-4}$, respectively. The transport equations for the renormalization group theory version of the $k-\varepsilon$ turbulence model can be found in any text on turbulent fluid mechanics such as Wilcox [12]. An initial set of grid independence studies was done to select a mesh that is adequate - judged by the smoothness of average surface temperature over a 24-hour period.

\section{RESULTS AND DISCUSSION}

The buildings are rotated as a block through an angle, $\theta$, of $360^{\circ}$, in the clockwise direction, around the $y$-axis, in increments of $10^{\circ}$. For each angle simulation, the time step size is one hour. The simulation starts at 5:00 $\mathrm{AM}$ and ends at 7:00 PM. In order to compare between several orientations of the buildings as a block, the average solar flux per orientation is obtained by taking its average over the whole 14-hour span. It is seen from the result that, in figure 3, the optimum orientation of the group of buildings is when the angle is zero, meaning the lone, offset building protrudes south from the other two. A comparable minimum point in terms of the total solar flux happens when the angle is 180 degrees, which is expected since the wind direction was fixed to north in all cases of this study.

To take a close look at each angle result, the area-weighted average of the solar flux as well as the areaweighted average of the temperature on each building are plotted against the hour of the day. Sample results for the (additional) case of $\theta=15^{\circ}$ are shown in figure 4 . The figure shows the correlation of the incidence of the total 
solar flux with the average temperatures of the buildings. It also shows some of the effect of the mutual shading provided by the one building to the rest.

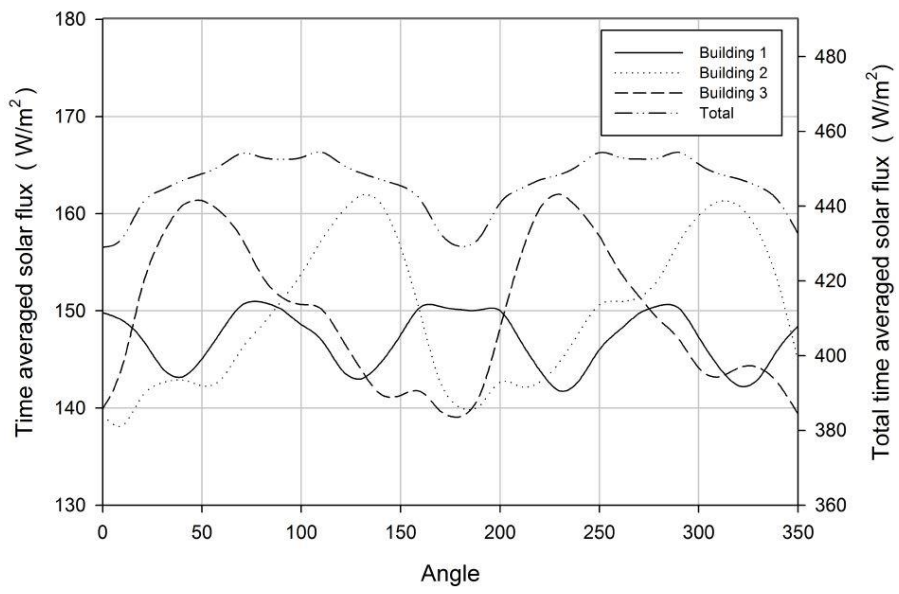

Figure 3. Total (Radiative + Convective) Heat Gain of Buildings Vs. Angle (Orientation)

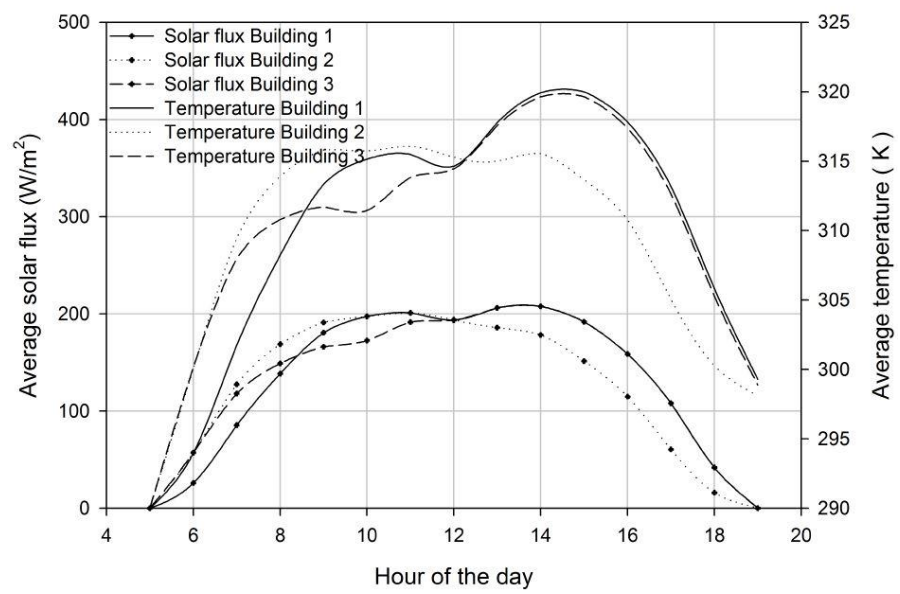

Figure 4. Average Surface Temperature and Solar Flux for the Buildings vs. Time of Day

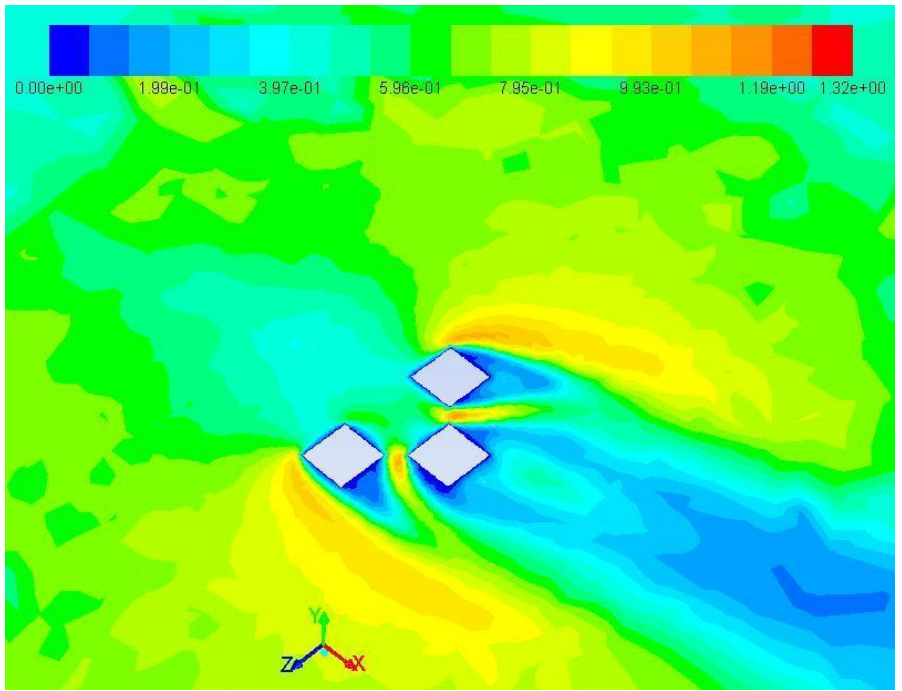

Figure 5. Sample Air Flow on A 1-M High Plane for the Case of $\Theta=0$, At 7:00 AM 
The air flow around the buildings are also shown in figure 5 at a height of $1 \mathrm{~m}$ above ground, for the case of $\theta=0^{\circ}$. The small effect of the convection could be seen when air carrying heat from one of the two buildings starts to sway in the direction of the protruding building. Equally important, since the ground is modeled (correctly) as a participating surface, this result suggests that the buildings, even if not mutually shaded exactly, decrease the load seen by other buildings when they help keep the temperature of the grounds from rising. Combined with the effect of the air breeze, the solar load carried by the buildings has a double benefit.

\section{CONCLUSION}

This study focuses on the optimum orientation of a group of buildings with respect to incident solar radiation. Such an optimization increases indoor comfort through minimizing glares and direct solar radiation, and reduces energy consumption. The geometry is constructed and meshed using Gambit and solved using the Fluent finite volume software. With both convection and radiation (direct and diffuse) considered, the total energy intake of the group of buildings had clear minimum and maximum.

Reported results show that a reduction in solar energy intake can be achieved when the buildings configuration is optimized. A maximum of $6 \%$ reduction in solar flux can be achieved by orienting the group of buildings at an angle of zero, i.e., with two buildings dead north of the third. The reduction is solar radiation intake depends on the building, with wind direction playing a small, but not negligible role. The study also suggests that such and similar building compounds could benefit from a radiation barrier that partially shields the south facing façades and a convection barrier reducing convection heat gain based on TYM-predicted data. All in all, it is shown that the reduction using solar optimization is significant, compared to optimizing the building morphology alone [13].

Ongoing work examines the effect of the sensitivity of these reductions in energy to wind direction and convection heat transfer, primarily by altering the wind strength and direction.

\section{NOMENCLATURE}

HVAC Heating Ventilating and Air-Conditioning

IR Infrared radiation

$\theta \quad$ Building rotation angle

\section{REFERENCES}

[1] Mohammed A. Al-Ajmi and Raed I. Bourisli. Optimum design of segmented passive-constrained layer damping treatment through genetic algorithms. Mechanics of Advanced Materials and Structures, 15(3):250-257, 2008 .

[2] Rongxin Yin, Peng Xu, and Pengyuan Shen. Case study: Energy savings from solar window film in two commercial buildings in Shanghai. Energy and Buildings, 45:132-140, 2012.

[3] Ronghui Qi, Lin Lu, and Hongxing Yang. Investigation

on air-conditioning load profile and energy consumption of desiccant cooling system for commercial buildings in Hong Kong. Energy and Buildings, 49:509-518, 2012.

[4] B. Ahmadkhani Maleki. Shading: Passive cooling an energy conservation in buildings. International Journal on Technical and Physical Problems of Engineering" (IJTPE), 3(4):72-79, 2012.

[5] Ahmed S. Muhaisen and Huda M. Abed. Effect of urban geometry and spacing on the thermal performance in the Mediterranean climate of the Gaza Strip. Journal of Architecture and Planning, 26(1):1-14, 2014.

[6] Carlo Ratti, Dana Raydan, and Koen Steemers. Building form and environmental performance: archetypes, analysis and an arid climate. Energy and Buildings, 35(7):49-59, 2003.

[7] J. Strømann-Andersen and P. A. Sattrup. Building form and environmental performance: archetypes, analysis and an arid climate. Energy and Buildings, 43(8):2011-2020, 2011.

[8] Viktor Dorer, Jonas Allegrini, Kristina Orehounig, Peter Moonen, Govinda Upadhyay, Jérôme Kämpf, and Jan Carmeliet. Modelling the urban microclimate and its impact on the energy demand of buildings and building clusters. In Proceedings of the 13th Conference of the International Building Performance Simulation Association, pages 3483-3489, Chambéry, France, August 2013.

[9] M. M. E. van Esch, R. H. J. Looman, and G. J. de Bruin-Hordijk. The effects of urban and building design parameters on solar access to the urban canyon and the potential for direct passive solar heating strategies. Energy and Buildings, 43:189-200, 2012.

[10]Limor Shashua-Bar, Yigal Tzamir, and Milo E. Homan. Thermal effects of building geometry and spacing on the urban canopy layer microclimate in a hot-humid climate in summer. International Journal of Climatology, 24(13):1729-1742, 2004. 
[11] E. Krüger and F. Rasia D. Pearlmutte. Evaluating the impact of canyon geometry and orientation on cooling loads in a high-mass building in a hot dry environment. Applied Energy, 87(6):2068-2078, 2010.

[12] D. C. Wilcox. Turbulence Modelling for CFD. DCW Industries Inc., La Canada, CA, USA, 1993.

[13] Raed I. Bourisli and Adnan A. AlAnzi. Closed form correlation of buildings energy use with key design parameters calibrated using a genetic algorithm. ASME Journal of Solar Energy Engineering, 133(4):041005-1-9, 2011. 